\title{
Lymphocyte count and platelet volume predicts postoperative complications in esophagectomy for cancer: a cohort study
}

\author{
Francisco TUSTUMI, Flávio Roberto TAKEDA, Antonio Adolfo Guerra Soares BRANDÃO, \\ Rubens Antonio Aissar SALLUM, Ulysses RIBEIRO JUNIOR and Ivan CECCONELLO
}

Received 28/6/2019

Accepted 15/8/2019

\begin{abstract}
Background - Biomarkers from routine complete blood count are known predictive factors of long-term outcomes in cancer patients. The value of these biomarkers in the setting of trimodal therapy for esophageal cancer in predicting early postoperative outcomes is not studied. Objective - The present study evaluated the value of cellular blood components changes during neoadjuvant chemoradiotherapy followed by curative intent esophagectomy for cancer in predicting postoperative mortality and morbidity. Methods - A cohort of 149 consecutive patients that underwent chemoradiotherapy using platinum- and taxane-based regimens followed by esophagectomy was analyzed. Cellular components of blood collected before neoadjuvant therapy (period A) and before surgery (period B) were assessed for postoperative mortality and complications. Univariate and multivariate Cox regression models were applied to evaluate the independent prognostic significance of blood count variables. Results - Postoperative morbidity was present in $46 \%$ of the patients. On multiple regression analysis platelet volume (B) (OR: $1.53 ; 95 \%$ CI: $1.2-2.33$ ) was an independent predictor of general complications. Severe postoperative surgical complications were present in $17 \%$ of the patients. On multiple regression analysis, lymphocyte decrease between B-A periods (OR: 0.992; 95\% CI: 0.990-0.997) was related to higher risk for severe complications. Cervical anastomotic leakage was present in $25.6 \%$ of the patients. On univariate analysis eosinophil count in A and B periods was related to cervical anastomotic leakage. For this outcome, multivariate joint model could not identify independent risk variables of cellular components of blood. The 30-day mortality rate was $7.4 \%$. On univariate analysis, platelet count in period B was associated to higher risk for mortality. The multivariate joint model could not accurately predict mortality due to the few number of patients in the mortality group. Conclusion - This is the first study to assess the relationship between peripheral blood count variables changes during neoadjuvant chemoradiotherapy using a platinum- and taxane-based regimen followed by curative intent esophagectomy for cancer in predicting postoperative complications. The platelet volume prior to surgery is related to postoperative complications and the lymphocyte count change prior to surgery predicts severe postoperative complications in the setting of trimodal therapy for esophageal cancer. HEADINGS - Esophageal neoplasms. Neoadjuvant therapy. Blood cells. Leukocytes. Blood platelets. Lymphocytes. Neutrophils.
\end{abstract}

\section{INTRODUCTION}

Esophageal cancer treatment is based on neoadjuvant chemoradiotherapy followed by surgery in most potentially curable esophageal cancer ${ }^{(1,2)}$. Esophagectomy usually presents high postoperative morbidity and mortality, and certain complications may even influence long-term survival ${ }^{(3)}$.

Accumulating evidence shows that preoperative hematological biomarkers from routine complete blood count, such as red cell distribution width (RDW), neutrophil-to-lymphocyte ratio (NLR), platelet-to-lymphocyte ratio (PLR), and mean corpuscular volume (MCV) may predict long-term outcomes in cancer ${ }^{(4-14)}$. For esophageal cancer, preoperative NLR before surgery predicts overall survival (OS) and disease-free survival (DFS) ${ }^{(15)}$. Postoperative NLR elevation after surgery may also predict early postoperative outcomes in esophageal cancer ${ }^{(16)}$.

The value of cellular blood components prior to neoadjuvant therapy and prior to surgery in the setting of trimodal therapy for esophageal cancer in predicting early postoperative complications and mortality has not been reported, and is the aim of this study.

\section{METHODS}

A retrospective cohort was performed, assessing the correlation of cellular components of blood and their changes during trimodal therapy for esophageal carcinoma with postoperative mortality and postoperative complications. Consecutive patients of a single institute with completion of neoadjuvant chemoradiotherapy using platinum- and taxane-based regimens, followed by curative intent esophagectomy, were selected. Peripheral blood was obtained in two different moments: before neoadjuvant therapy (A); and before surgery (1-7 days) (B). Blood test was not collected during acute infectious disease, neither during targeted antitumor therapy.

Recruitment included 2009 to 2019 period. Patients were staged with endoscopy, CT-scan, and PET-scan prior to neoadjuvant therapy and classified accordingly to the 8th edition of UICC staging ${ }^{(17)}$. Patients were followed with clinical evaluation, peripheral blood analysis, CT-scan and endoscopy. Complications were classified accordingly to the international consensus on standardization of data collection for complications associated 
with esophagectomy ${ }^{(18)}$ and Clavien-Dindo classification ${ }^{(19)}$. Pathological response to neoadjuvant therapy was graded accordingly to Ryan score ${ }^{(20)}$. The local ethics committee approved the study.

\section{Statistical analysis}

For absolute and relative variable, chi-square test, Fisher test or likelihood-ratio test were used for each outcome. Multivariate Cox proportional hazard analysis was performed to determine independent risk factors for the outcomes. Only variables that were significant $(P<0.1)$ on univariate analysis were included as co-variables in the multivariable analyses. Data were assessed with IBM-SPSS software version 20.0, and a significance level of 0.05 was adopted.

\section{Ethics approval and consent to participate}

This study was approved by local Ethics Committee (CAPEPesq).

\section{RESULTS}

\section{Patients' baseline characteristics}

One hundred and forty-nine consecutive patients underwent neoadjuvant chemoradiotherapy using platinum- and taxanebased regimens followed by esophagectomy and were included. The mean age was 60.9 years $(\mathrm{SD} \pm 8.6)$, with male predominance (75.8\%). There were 117 transthoracic (video-assisted thoracoscopic) procedures and 32 transhiatal procedures, all of them with cervical anastomosis. The median time from completion of neoadjuvant chemoradiotherapy to surgery was 13 weeks (IQR $8)$. The two chemotherapy regimens adopted were carboplatin and paclitaxel $(75.2 \%)$, and cisplatin and paclitaxel $(24.8 \%)$. The radiation therapy dosage was $41.4 \mathrm{cGy}(75 \%), 45 \mathrm{cGy}(13.5 \%)$, and $50.4 \mathrm{cGy}(11.5 \%)$.

\section{General postoperative complications}

The postoperative morbidity was present in $46 \%$ of the patients. Clinical postoperative complications, such as pneumonia, cardiovascular events, respiratory failure, thromboembolic events, were present in $22 \%$ of the patients. Surgical complications, such as bleeding, chylothorax, cervical anastomotic leak, were present in $35.5 \%$ of the patients.

On univariate analysis age was related to general postoperative complications. Before surgery (period B), platelet volume was also related to general complications. On multiple regression analysis, age (OR: 1.05; 95\% CI: 1.01-1.1; $P=0.019)$ and platelet volume (B) (OR: $1.53 ; 95 \% \mathrm{CI}: 1.2-2.33 ; P=0.045)$ were independent predictors of general complications (TABLE 1 and FIGURE 1). The predictive model for general complications can be expressed by the following equation: equation $=\exp (-7.3+0.049 \times$ Age +0.423 $\mathrm{x}$ Platelet Volume (B)) / $(1+\exp (-7.3+0.049 \mathrm{x}$ Age $+0.423 \mathrm{x}$ Platelet Volume (B))).

\section{Severe postoperative complications}

Severe postoperative complications (Clavien-Dindo $\geq$ IIIa) were present in $17 \%$ of the patients.

On univariate analysis transhiatal access was related to severe postoperative complications. The decrease of lymphocytes between B-A periods was correlated to severe complications. On multiple regression analysis, surgical access (thoracoscopy: OR: 0.23; $95 \%$ CI: $0.088-0.602 ; P=0.003)$ and lymphocyte change between $\mathrm{B}-\mathrm{A}$ periods (OR: $0.992 ; 95 \% \mathrm{CI}$ : $0.990-0.997 ; P=0.046)$ were related to higher risk for severe complications (TABLE 2 and FIGURE 2).
The predictive model for severe complications can be expressed by the following equation $=\exp (-1.12-0.001 \mathrm{x}$ Lymphocyte (B-A) -1.47 (if thoracoscopy)) / (1+exp $(-1.12-0.001$ x Lymphocyte (B-A) -1.47 (if thoracoscopy)).

\section{Cervical anastomotic leakage}

Cervical anastomotic leakage was present in $25.6 \%$ of the patients, and mostly was easily manageable and classified as grade ${ }^{(18)}$ I or II $(90 \%)$

On univariate analysis grade of cellular differentiation was related to cervical anastomotic leakage. Eosinophil count in A (OR: $1.001 ; 95 \%$ CI: 1-1.002; $P=0.011$ ), and B (OR: $1.001 ; 95 \%$ CI: $0.999-1.002 ; P=0.038)$ periods was also related to cervical anastomotic leakage (TABLE 3). The multivariate joint model was performed and the risk for cervical anastomotic leakage was constant, independently of the blood cellular variables.

\section{Postoperative mortality}

The 30-day mortality rate was 7.4\%.

On univariate analysis, age, histology, clinical stage, Ryan pathological response score, and complications were related to 30 -days postoperartive mortality. For cellular components of blood, all variables were not related to postoperartive mortality, but platelet count in period B (OR: 1.007; 95\% CI: 0.999-1.017; $P=0.017$ ) (TABLE 4). The multivariate joint model could not accurately predict postoperative 30 -day mortality due to the few number of patients in the mortality group.

\section{DISCUSSION}

Results of this cohort of 149 consecutive esophageal cancer patients that underwent neoadjuvant chemoradiotherapy using a platinum- and taxane-based regimen followed by curative intent esophagectomy suggest that mean platelet volume and lymphocyte decrease during neoadjuvant therapy are independent variables that can predict postoperative complications.

It has been well established that there is a complex relationship among tumor, systemic inflammation, and nutritional and immune status $^{(5,11,21-23)}$ that may influence in surgical complications risks ${ }^{(24-26)}$.

Cancer cells release growth factors and inflammatory mediators that stimulate production and activation of peripheral blood platelets $^{(27,28)}$. Increased mean platelet volume is an early index of activated platelets ${ }^{(29)}$. In our study, mean platelet volume was related to higher risk for general complications, although the peripheral platelet count was not related to higher risk for complications.

Lymphocyte decrease in cancer patients reflects host immune function due to hormonal change, malnutrition, antitumor immune response, or may be due to chemotherapy induced depletion ${ }^{(30-36)}$. Lymphopenia has strong relationship with lower heart ejection fraction postoperatively ${ }^{(37)}$, major adverse cardiac outcomes ${ }^{(38-40)}$ and infectious complications ${ }^{(36)}$. In the present study, lymphocyte decrease between B and A periods increases the risk for severe postoperative complications. Despite lymphopenia may also reflect radiation therapy side effect ${ }^{(41)}$, in the present radiation dose did not influence morbidity.

Although previous studies reported that preoperative NLR might predict severe postoperative complications for colorectal, head and neck, and gastric surgery ${ }^{(26,42-46)}$, in the present study NLR and PLR were not related to postoperative complications in esophageal carcinoma. 
TABLE 1. General postoperative complications. Univariate analysis. Cellular components of blood are expressed in mean \pm standard deviation (SD).

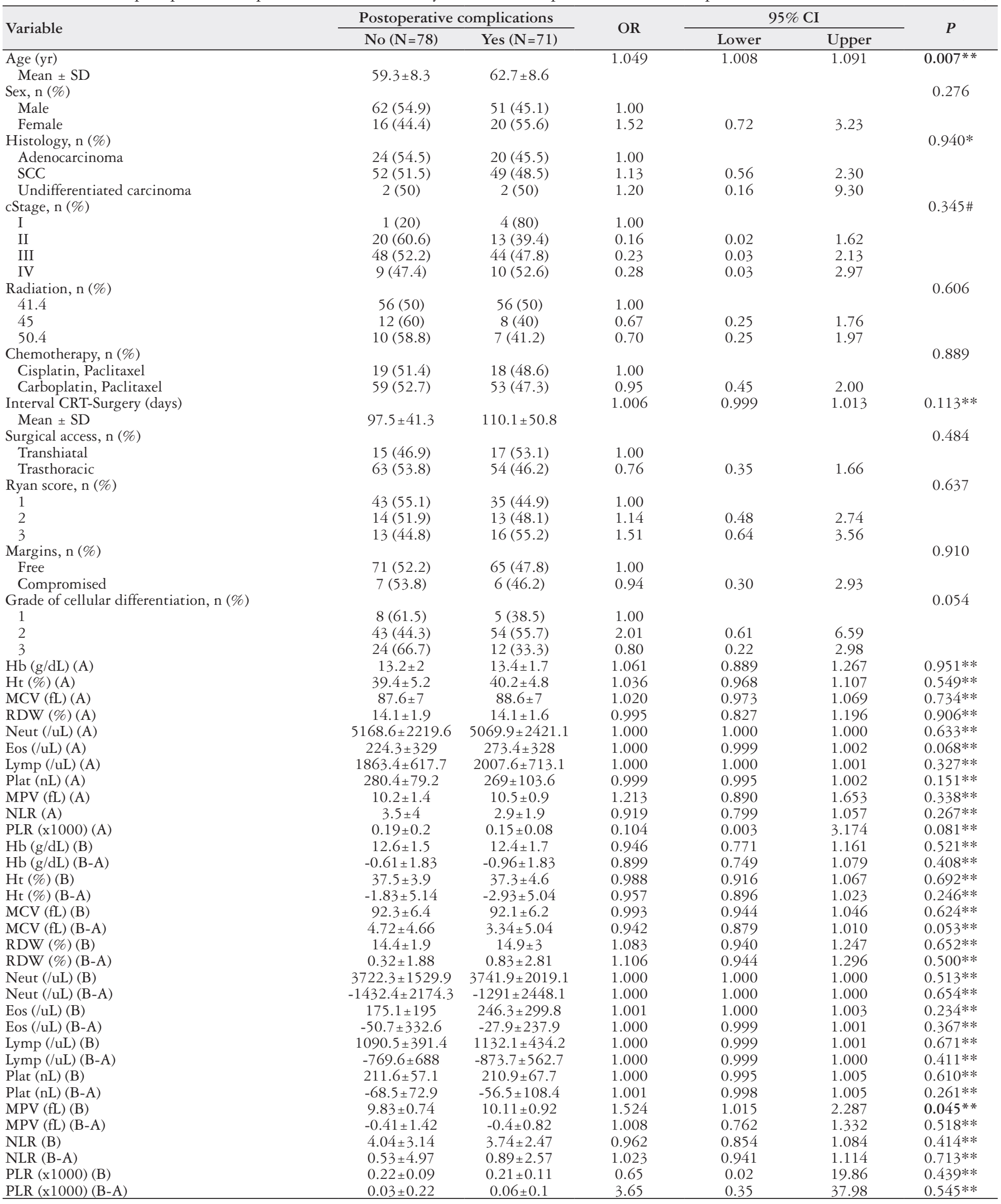

(A): before neoadjuvant therapy; (B): before surgery. Chi-squared test; *Fisher's exact test; \#Likelihood ratio test; ** Mann-Whitney test. 
TABLE 2. Clavien-Dindo classification of surgical complications. Univariate analysis. Cellular components of blood are expressed in mean \pm standard deviation (SD).

\begin{tabular}{|c|c|c|c|c|c|c|}
\hline \multirow{2}{*}{ Variable } & \multicolumn{2}{|c|}{ Clavien-Dindo } & \multirow{2}{*}{ OR } & \multicolumn{2}{|c|}{$95 \% \mathrm{CI}$} & \multirow{2}{*}{$P$} \\
\hline & $<$ IIIa $(\mathrm{N}=122)$ & $\geq \operatorname{IIIa}(\mathrm{N}=27)$ & & Lower & Upper & \\
\hline Age (yr) & & & 1.033 & 0.982 & 1.088 & $0.314 * *$ \\
\hline Mean \pm SD & $60.5 \pm 8.5$ & $62.8 \pm 8.7$ & & & & \\
\hline Sex, n $(\%)$ & & & & & & 0.463 \\
\hline Male & $94(83.2)$ & $19(16.8)$ & 1.00 & & & \\
\hline Female & $28(77.8)$ & $8(22.2)$ & 1.41 & 0.56 & 3.57 & \\
\hline Histology, $\mathrm{n}(\%)$ & & & & & & $0.197 \#$ \\
\hline Adenocarcinoma & $33(75)$ & $11(25)$ & 1.00 & & & \\
\hline SCC & $85(84.2)$ & $16(15.8)$ & 0.57 & 0.24 & 1.34 & \\
\hline Undifferentiated carcinoma & $4(100)$ & $0(0)$ & $\&$ & & & \\
\hline cStage, n $(\%)$ & & & & & & $0.151 \#$ \\
\hline 1 & $5(100)$ & $0(0)$ & 1.00 & & & \\
\hline 2 & $30(90.9)$ & $3(9.1)$ & $\&$ & & & \\
\hline 3 & $73(79.3)$ & $19(20.7)$ & $\&$ & & & \\
\hline 4 & $14(73.7)$ & $5(26.3)$ & $\&$ & & & \\
\hline Radiation, n (\%) & & & & & & $0.575 \#$ \\
\hline 41.4 & $92(82.1)$ & $20(17.9)$ & 1.00 & & & \\
\hline 45 & $15(75)$ & $5(25)$ & 1.53 & 0.50 & 4.71 & \\
\hline 50.4 & $15(88.2)$ & $2(11.8)$ & 0.61 & 0.13 & 2.90 & \\
\hline Chemotherapy, n (\%) & & & & & & 0.105 \\
\hline Cisplatin, Paclitaxel & $27(73)$ & $10(27)$ & 1.00 & & & \\
\hline Carboplatin, Paclitaxel & $95(84.8)$ & $17(15.2)$ & 0.48 & 0.20 & 1.18 & \\
\hline Interval CRT-Surgery (days) & & & 1.007 & 0.999 & 1.015 & $0.099 * *$ \\
\hline Mean \pm SD & $100.5 \pm 44$ & $117.1 \pm 54.4$ & & & & \\
\hline Surgical access, n (\%) & & & & & & 0.007 \\
\hline Transhiatal & $21(65.6)$ & $11(34.4)$ & 1.00 & & & \\
\hline Trasthoracic & $101(86.3)$ & $16(13.7)$ & 0.30 & 0.12 & 0.74 & \\
\hline Ryan score, n (\%) & & & & & & 0.775 \\
\hline 1 & $65(83.3)$ & $13(16.7)$ & 1.00 & & & \\
\hline 2 & $21(77.8)$ & $6(22.2)$ & 1.43 & 0.48 & 4.23 & \\
\hline 3 & $23(79.3)$ & $6(20.7)$ & 1.30 & 0.44 & 3.83 & \\
\hline Margins, n (\%) & & & & & & $0.464 *$ \\
\hline Free & $110(80.9)$ & $26(19.1)$ & 1.00 & & & \\
\hline Compromised & $12(92.3)$ & $1(7.7)$ & 0.35 & 0.04 & 2.83 & \\
\hline Grade of cellular differentiation, $\mathrm{n}(\%)$ & & & & & & $0.633 \#$ \\
\hline 1 & $11(84.6)$ & $2(15.4)$ & 1.00 & & & \\
\hline 2 & $77(79.4)$ & $20(20.6)$ & 1.43 & 0.29 & 6.97 & \\
\hline 3 & $31(86.1)$ & $5(13.9)$ & 0.89 & 0.15 & 5.25 & \\
\hline $\mathrm{Hb}(\mathrm{g} / \mathrm{dL})(\mathrm{A})$ & $13.3 \pm 1.9$ & $13.2 \pm 1.4$ & 0.971 & 0.778 & 1.212 & $0.349 * *$ \\
\hline $\mathrm{Ht}(\%)(\mathrm{A})$ & $39.8 \pm 5.2$ & $39.8 \pm 3.8$ & 1.000 & 0.919 & 1.088 & $0.717 * *$ \\
\hline MCV (fL) (A) & $88 \pm 7$ & $88.4 \pm 6.8$ & 1.007 & 0.947 & 1.069 & $0.974 * *$ \\
\hline RDW $(\%)(A)$ & $14 \pm 1.9$ & $14.2 \pm 1.3$ & 1.048 & 0.842 & 1.305 & $0.195 * *$ \\
\hline Neut (/uL) (A) & $5088.8 \pm 2316.5$ & $5263.3 \pm 2328.3$ & 1.000 & 1.000 & 1.000 & $0.695 * *$ \\
\hline $\operatorname{Eos}(/ \mathrm{uL})(\mathrm{A})$ & $251.3 \pm 351$ & $233.3 \pm 203.1$ & 1.000 & 0.998 & 1.001 & $0.583 * *$ \\
\hline $\operatorname{Lymp}(/ \mathrm{uL})(\mathrm{A})$ & $1886.4 \pm 650.4$ & $2140.4 \pm 712.6$ & 1.001 & 1.000 & 1.001 & $0.102 * *$ \\
\hline Plat (nL) (A) & $276.9 \pm 92.5$ & $266 \pm 88.3$ & 0.999 & 0.994 & 1.004 & $0.498 * *$ \\
\hline MPV (fL) (A) & $10.4 \pm 1.2$ & $10.3 \pm 0.9$ & 0.956 & 0.682 & 1.338 & $0.509 * *$ \\
\hline $\operatorname{NLR}(\mathrm{A})$ & $3.3 \pm 3.4$ & $2.8 \pm 2.1$ & 0.934 & 0.765 & 1.140 & $0.280 * *$ \\
\hline $\operatorname{PLR}(\mathrm{x} 1000)(\mathrm{A})$ & $0.18 \pm 0.17$ & $0.14 \pm 0.08$ & 0.020 & 0.000 & 11.410 & $0.058 * *$ \\
\hline $\mathrm{Hb}(\mathrm{g} / \mathrm{dL})(\mathrm{B})$ & $12.5 \pm 1.6$ & $12.6 \pm 1.7$ & 1.024 & 0.786 & 1.335 & $0.821 * *$ \\
\hline $\mathrm{Hb}(\mathrm{g} / \mathrm{dL})(\mathrm{B}-\mathrm{A})$ & $-0.81 \pm 1.86$ & $-0.65 \pm 1.71$ & 1.049 & 0.836 & 1.315 & $0.493 * *$ \\
\hline $\mathrm{Ht}(\%)(\mathrm{B})$ & $37.4 \pm 4.2$ & $37.7 \pm 4.7$ & 1.019 & 0.923 & 1.125 & $0.747 * *$ \\
\hline $\mathrm{Ht}(\%)(\mathrm{B}-\mathrm{A})$ & $-2.42 \pm 5.23$ & $-2.08 \pm 4.57$ & 1.013 & 0.934 & 1.099 & $0.637 * *$ \\
\hline MCV (fL) (B) & $92.1 \pm 6.4$ & $92.7 \pm 6.1$ & 1.016 & 0.950 & 1.087 & $0.770 * *$ \\
\hline MCV (fL) (B-A) & $3.99 \pm 4.94$ & $4.37 \pm 4.66$ & 1.016 & 0.932 & 1.107 & $0.980 * *$ \\
\hline RDW $(\%)(B)$ & $14.7 \pm 2.6$ & $14.7 \pm 2.1$ & 1.007 & 0.854 & 1.188 & $0.882 * *$ \\
\hline RDW (\%) (B-A) & $0.58 \pm 2.5$ & $0.5 \pm 1.76$ & 0.985 & 0.821 & 1.182 & $0.803 * *$ \\
\hline Neut (/uL) (B) & $3689.6 \pm 1678.7$ & $3919.6 \pm 2168.1$ & 1.000 & 1.000 & 1.000 & $0.887 * *$ \\
\hline Neut (/uL) (B-A) & $-1369.3 \pm 2226.3$ & $-1343.7 \pm 2660$ & 1.000 & 1.000 & 1.000 & $0.912 * *$ \\
\hline $\operatorname{Eos}(/ \mathrm{uL})(\mathrm{B})$ & $209.8 \pm 247.5$ & $204.1 \pm 274.7$ & 1.000 & 0.998 & 1.002 & $0.778 * *$ \\
\hline $\operatorname{Eos}(/ \mathrm{uL})(\mathrm{B}-\mathrm{A})$ & $-42.1 \pm 292.2$ & $-29.3 \pm 287$ & 1.000 & 0.999 & 1.002 & $0.289 * *$ \\
\hline Lymp (/uL) (B) & $1101.7 \pm 394.9$ & $1148.2 \pm 484.5$ & 1.000 & 0.999 & 1.001 & $0.972 * *$ \\
\hline Lymp (/uL) (B-A) & $-780.3 \pm 627.3$ & $-992.2 \pm 630.2$ & 0.999 & 0.999 & 1.000 & $0.049 * *$ \\
\hline Plat (nL) (B) & $213.6 \pm 62$ & $200.3 \pm 62.2$ & 0.996 & 0.989 & 1.004 & $0.243 * *$ \\
\hline Plat (nL) (B-A) & $-62.1 \pm 94.7$ & $-65.7 \pm 76$ & 1.000 & 0.995 & 1.004 & $0.945 * *$ \\
\hline MPV (fL) (B) & $9.93 \pm 0.83$ & $10.1 \pm 0.88$ & 1.269 & 0.780 & 2.067 & $0.462 * *$ \\
\hline MPV (fL) (B-A) & $-0.46 \pm 1.24$ & $-0.2 \pm 0.78$ & 1.160 & 0.854 & 1.576 & $0.069 * *$ \\
\hline $\operatorname{NLR}(\mathrm{B})$ & $3.94 \pm 2.96$ & $3.71 \pm 2.27$ & 0.970 & 0.824 & 1.142 & $0.839 * *$ \\
\hline NLR (B-A) & $0.66 \pm 4.2$ & $0.88 \pm 2.98$ & 1.015 & 0.909 & 1.133 & $0.846 * *$ \\
\hline PLR (x1000) (B) & $0.22 \pm 0.1$ & $0.2 \pm 0.09$ & 0.106 & 0.001 & 15.242 & $0.337 * *$ \\
\hline PLR (x1000) (B-A) & $0.04 \pm 0.19$ & $0.06 \pm 0.1$ & 2.106 & 0.102 & 43.565 & $0.757 * *$ \\
\hline
\end{tabular}

(A): before neoadjuvant therapy; (B): before surgery. Chi-squared test; *Fisher's exact test; \#Likelihood ratio test; ** Mann-Whitney test. 
TABLE 3. Cervical anastomotic leakage. Univariate analysis. Cellular components of blood are expressed in mean \pm standard deviation (SD).

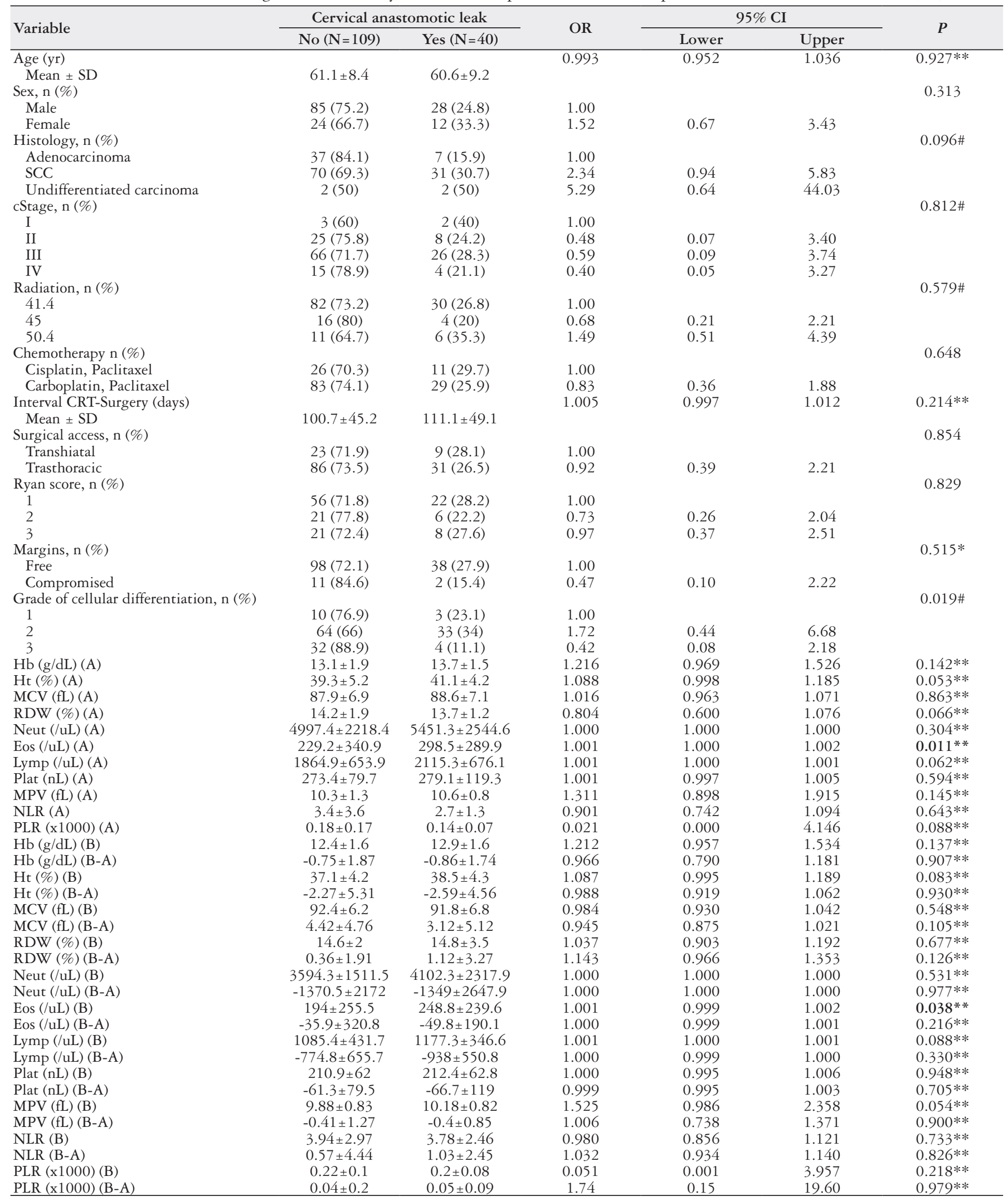

(A): before neoadjuvant therapy; (B): before surgery. Chi-squared test; *Fisher's exact test; \#Likelihood ratio test; ** Mann-Whitney test. 
TABLE 4. Postoperative mortality (30-days). Univariate analysis. Cellular components of blood are expressed in mean \pm standard deviation (SD).

\begin{tabular}{|c|c|c|c|c|c|c|c|}
\hline \multirow{2}{*}{ Variable } & \multicolumn{2}{|c|}{ Mortality (30-days) } & \multirow{2}{*}{ Total $(\mathrm{N}=149)$} & \multirow{2}{*}{ OR } & \multicolumn{2}{|c|}{$95 \% \mathrm{CI}$} & $D$ \\
\hline & $\mathrm{No}(\mathrm{N}=138)$ & Yes $(\mathrm{N}=11)$ & & & Lower & Upper & $P$ \\
\hline $\begin{array}{l}\text { Age }(y r) \\
\text { Mean } \pm \text { SD }\end{array}$ & $60.5 \pm 8.4$ & $66.9 \pm 8.6$ & $60.9 \pm 8.6$ & 1.113 & 1.017 & 1.218 & $0.034 * *$ \\
\hline Sex, n $(\%)$ & & & & & & & $0.462 *$ \\
\hline Male & $106(93.8)$ & $7(6.2)$ & 113 & 1.00 & & & \\
\hline Female & $32(88.9)$ & $4(11.1)$ & 36 & 1.89 & 0.52 & 6.88 & \\
\hline Histology, n (\%) & & & & & & & $0.043 \#$ \\
\hline Adenocarcinoma & $37(84.1)$ & $7(15.9)$ & 44 & 1.00 & & & \\
\hline SCC & $97(96)$ & $4(4)$ & 101 & 0.22 & 0.06 & 0.79 & \\
\hline Undifferentiated carcinoma & $4(100)$ & $0(0)$ & 4 & $\&$ & & & \\
\hline cStage, n (\%) & & & & & & & $0.011 \#$ \\
\hline I & $5(100)$ & $0(0)$ & 5 & 1.00 & & & \\
\hline II & $33(100)$ & $0(0)$ & 33 & $\&$ & & & \\
\hline III & $81(88)$ & $11(12)$ & 92 & $\&$ & & & \\
\hline IV & $19(100)$ & $0(0)$ & 19 & $\&$ & & & \\
\hline Radiation, n (\%) & & & & & & & $0.882 \#$ \\
\hline 41.4 & $104(92.9)$ & $8(7.1)$ & 112 & 1.00 & & & \\
\hline 45 & $18(90)$ & $2(10)$ & 20 & 1.44 & 0.28 & 7.36 & \\
\hline 50.4 & $16(94.1)$ & $1(5.9)$ & 17 & 0.81 & 0.10 & 6.94 & \\
\hline Chemotherapy, n (\%) & & & & & & & $>0.999 *$ \\
\hline Cisplatin, Paclitaxel & $34(91.9)$ & $3(8.1)$ & 37 & 1.00 & & & \\
\hline Carboplatin, Paclitaxel & $104(92.9)$ & $8(7.1)$ & 112 & 0.87 & 0.22 & 3.47 & \\
\hline Interval CRT-Surgery (days) & & & & 1.011 & 1.000 & 1.022 & $0.107 * *$ \\
\hline Mean \pm SD & $101.3 \pm 43.6$ & $130.8 \pm 70$ & $103.5 \pm 46.3$ & & & & \\
\hline Surgical access, n (\%) & & & & & & & $0.251 *$ \\
\hline Transhiatal & $28(87.5)$ & $4(12.5)$ & 32 & 1.00 & & & \\
\hline Trasthoracic & $110(94)$ & $7(6)$ & 117 & 0.45 & 0.12 & 1.63 & \\
\hline Ryan score, n (\%) & & & & & & & $0.024 \#$ \\
\hline 1 & $75(96.2)$ & $3(3.8)$ & 78 & 1.00 & & & \\
\hline 2 & $21(77.8)$ & $6(22.2)$ & 27 & 7.14 & 1.65 & 31.00 & \\
\hline 3 & $27(93.1)$ & $2(6.9)$ & 29 & 1.85 & 0.29 & 11.69 & \\
\hline Margins, n (\%) & & & & & & & $>0.999 *$ \\
\hline Free & $126(92.6)$ & $10(7.4)$ & 136 & 1.00 & & & \\
\hline Compromised & $12(92.3)$ & $1(7.7)$ & 13 & 1.05 & 0.12 & 8.92 & \\
\hline Grade of cellular differentiation, n (\%) & & & & & & & $0.865 \#$ \\
\hline 1 & $12(92.3)$ & $1(7.7)$ & 13 & 1.00 & & & \\
\hline $\begin{array}{l}2 \\
3\end{array}$ & $89(91.8)$ & $8(8.2)$ & 97 & 1.08 & 0.12 & 9.40 & \\
\hline 3 & $34(94.4)$ & $2(5.6)$ & 36 & 0.71 & 0.06 & 8.51 & \\
\hline Postoperative complications, n (\%) & & & & & & & 0.003 \\
\hline No & $77(98.7)$ & $1(1.3)$ & 78 & 1.00 & & & \\
\hline Yes & $61(85.9)$ & $10(14.1)$ & 71 & 12.62 & 1.57 & 101.33 & \\
\hline Cervical anastomotic leak, n (\%) & & & & & & & $0.728 *$ \\
\hline No & $100(91.7)$ & $9(8.3)$ & 109 & 1.00 & & & \\
\hline Yes & $38(95)$ & $2(5)$ & 40 & 0.59 & 0.12 & 2.83 & \\
\hline Clavien Dindo, n (\%) & & & & & & & $<0.001 *$ \\
\hline$<$ IIIa & $121(99.2)$ & $1(0.8)$ & 122 & 1.00 & & & \\
\hline$\geq$ IIIa & $17(63)$ & $10(37)$ & 27 & 71.18 & 8.57 & 591.43 & \\
\hline $\mathrm{Hb}(\mathrm{g} / \mathrm{dL})(\mathrm{A})$ & $13.3 \pm 1.9$ & $12.7 \pm 1.5$ & $13.3 \pm 1.9$ & 0.847 & 0.636 & 1.129 & $0.103 * *$ \\
\hline $\mathrm{Ht}(\%)(\mathrm{A})$ & $39.9 \pm 5$ & $38.2 \pm 4.5$ & $39.8 \pm 5$ & 0.939 & 0.843 & 1.047 & $0.177 * *$ \\
\hline $\operatorname{MCV}(\mathrm{fL})(\mathrm{A})$ & $88 \pm 6.9$ & $89.6 \pm 7.5$ & $88.1 \pm 7$ & 1.036 & 0.944 & 1.137 & $0.604 * *$ \\
\hline RDW $(\%)(A)$ & $14.1 \pm 1.8$ & $14.1 \pm 1.1$ & $14.1 \pm 1.8$ & 1.005 & 0.712 & 1.419 & $0.450 * *$ \\
\hline Neut (/uL) (A) & $5086.7 \pm 2319.8$ & $5543.6 \pm 2270.8$ & $5120.9 \pm 2311.6$ & 1.000 & 1.000 & 1.000 & $0.515 * *$ \\
\hline $\operatorname{Eos}(/ \mathrm{uL})(\mathrm{A})$ & $253 \pm 336$ & $186.4 \pm 212.7$ & $248 \pm 328.3$ & 0.999 & 0.996 & 1.002 & $0.443 * *$ \\
\hline $\operatorname{Lymp}(/ \mathrm{uL})(\mathrm{A})$ & $1926.8 \pm 633$ & $2010.5 \pm 1039.9$ & $1933 \pm 667.1$ & 1.000 & 0.999 & 1.001 & $0.860 * *$ \\
\hline Plat (nL) (A) & $275.6 \pm 94.1$ & $266.3 \pm 46.5$ & $274.9 \pm 91.6$ & 0.999 & 0.991 & 1.006 & $0.911 * *$ \\
\hline $\operatorname{MPV}(\mathrm{fL})(\mathrm{A})$ & $10.4 \pm 1.2$ & $10.3 \pm 0.6$ & $10.4 \pm 1.2$ & 0.959 & 0.589 & 1.560 & $0.667 * *$ \\
\hline NLR (A) & $3.1 \pm 3$ & $4.3 \pm 4.8$ & $3.2 \pm 3.2$ & 1.074 & 0.949 & 1.216 & $0.866 * *$ \\
\hline PLR (x1000) (A) & $0.17 \pm 0.15$ & $0.22 \pm 0.23$ & $0.17 \pm 0.15$ & 3.387 & 0.245 & 46.9 & $0.852 * *$ \\
\hline $\mathrm{Hb}(\mathrm{g} / \mathrm{dL})(\mathrm{B})$ & $12.5 \pm 1.5$ & $12.3 \pm 2$ & $12.5 \pm 1.6$ & 0.918 & 0.621 & 1.357 & $0.778 * *$ \\
\hline $\mathrm{Hb}(\mathrm{g} / \mathrm{dL})(\mathrm{B}-\mathrm{A})$ & $-0.81 \pm 1.82$ & $-0.36 \pm 2.02$ & $-0.78 \pm 1.83$ & 1.136 & 0.826 & 1.562 & $0.329 * *$ \\
\hline $\mathrm{Ht}(\%)(\mathrm{B})$ & $37.5 \pm 4.1$ & $37.2 \pm 5.9$ & $37.4 \pm 4.3$ & 0.984 & 0.852 & 1.138 & $0.849 * *$ \\
\hline $\mathrm{Ht}(\%)(\mathrm{B}-\mathrm{A})$ & $-2.47 \pm 5.06$ & $-0.98 \pm 5.72$ & $-2.36 \pm 5.11$ & 1.055 & 0.943 & 1.180 & $0.337 * *$ \\
\hline MCV (fL) (B) & $92.2 \pm 6.3$ & $92.5 \pm 6.4$ & $92.2 \pm 6.3$ & 1.008 & 0.913 & 1.112 & $0.942 * *$ \\
\hline $\operatorname{MCV}(\mathrm{fL})(\mathrm{B}-\mathrm{A})$ & $4.16 \pm 4.93$ & $2.91 \pm 4.24$ & $4.06 \pm 4.88$ & 0.948 & 0.835 & 1.077 & $0.467 * *$ \\
\hline RDW $(\%)(B)$ & $14.6 \pm 2.6$ & $15 \pm 1.6$ & $14.7 \pm 2.5$ & 1.056 & 0.862 & 1.293 & $0.219 * *$ \\
\hline RDW $(\%)(B-A)$ & $0.54 \pm 2.42$ & $0.96 \pm 1.83$ & $0.57 \pm 2.38$ & 1.064 & 0.861 & 1.315 & $0.339 * *$ \\
\hline Neut $(/ \mathrm{uL})(\mathrm{B})$ & $3704.9 \pm 1763.7$ & $4063.6 \pm 1927.9$ & $3731.6 \pm 1771.9$ & 1.000 & 1.000 & 1.000 & $0.532 * *$ \\
\hline Neut (/uL) (B-A) & $-1355.2 \pm 2305.7$ & $-1480 \pm 2372.3$ & $-1364.6 \pm 2302.7$ & 1.000 & 1.000 & 1.000 & $0.630 * *$ \\
\hline $\operatorname{Eos}(/ \mathrm{uL})(\mathrm{B})$ & $206.3 \pm 239.2$ & $240 \pm 389.7$ & $208.8 \pm 251.7$ & 1.000 & 0.998 & 1.003 & $0.852 * *$ \\
\hline Eos (/uL) (B-A) & $-47.3 \pm 279.4$ & $53.6 \pm 407.2$ & $-39.7 \pm 290.3$ & 1.002 & 0.999 & 1.004 & $0.476 * *$ \\
\hline $\operatorname{Lymp}(/ \mathrm{uL})(\mathrm{B})$ & $1089.9 \pm 380$ & $1363.6 \pm 668.7$ & $1110.2 \pm 411.3$ & 1.001 & 1.000 & 1.003 & $0.281 * *$ \\
\hline Lymp (/uL) (B-A) & $-833.6 \pm 600.3$ & $-646.8 \pm 953.7$ & $-819.5 \pm 631.1$ & 1.000 & 0.999 & 1.001 & $0.654 * *$ \\
\hline Plat (nL) (B) & $208.9 \pm 62.8$ & $243.1 \pm 40.9$ & $211.3 \pm 62$ & 1.007 & 0.999 & 1.017 & $0.017 * *$ \\
\hline Plat (nL) (B-A) & $-65.7 \pm 93.4$ & $-23.2 \pm 42.5$ & $-62.8 \pm 91.4$ & 1.007 & 0.998 & 1.017 & $0.058 * *$ \\
\hline MPV (fL) (B) & $9.97 \pm 0.83$ & $9.87 \pm 0.95$ & $9.96 \pm 0.84$ & 0.870 & 0.408 & 1.854 & $0.282 * *$ \\
\hline MPV (fL) (B-A) & $-0.41 \pm 1.2$ & $-0.43 \pm 0.76$ & $-0.41 \pm 1.17$ & 0.984 & 0.568 & 1.706 & $0.837 * *$ \\
\hline NLR (B) & $3.94 \pm 2.9$ & $3.38 \pm 2$ & $3.89 \pm 2.84$ & 0.907 & 0.672 & 1.223 & $0.504 * *$ \\
\hline NLR (B-A) & $0.84 \pm 3.87$ & $-0.97 \pm 5.21$ & $0.7 \pm 3.99$ & 0.925 & 0.826 & 1.035 & $0.352 * *$ \\
\hline PLR (x1000) (B) & $0.21 \pm 0.09$ & $0.23 \pm 0.12$ & $0.21 \pm 0.1$ & 4.632 & 0.010 & 2202.1 & $0.752 * *$ \\
\hline PLR $(x 1000)(B-A)$ & $0.05 \pm 0.17$ & $0.01 \pm 0.25$ & $0.04 \pm 0.17$ & 0.436 & 0.029 & 6.669 & $0.851 * *$ \\
\hline
\end{tabular}

(A): before neoadjuvant therapy; (B): before surgery. Chi-squared test; *Fisher's exact test; \#Likelihood ratio test; ** Mann-Whitney test. 


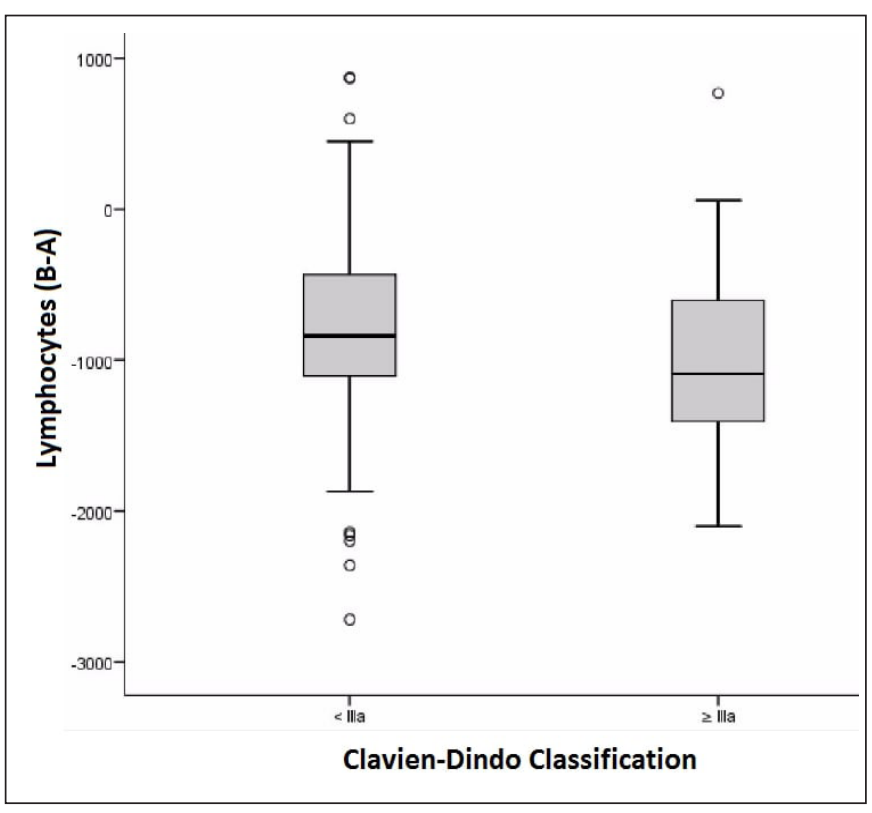

FIGURE 1. Box-plot of lymphocyte change between before surgery period (B) and before neoadjuvant therapy period (A), accordingly to ClavienDindo classification. Lymphocytes are expressed in units $/ \mu \mathrm{L}$.

The role of eosinophils in solid tumors is unclear, and may favor an antitumor inflammatory response, depending on the surrounding stimuli ${ }^{(47-50)}$. Eosinophils also might contribute to thrombophilia in patients with cancer ${ }^{(51,52)}$. In the present study, higher eosinophil level, either prior to neoadjuvant therapy or prior to surgery, was related to higher chance of esophageal anastomotic leak on univariate analysis. The effect was not retained on multivariate analysis. This may be attributable to the small sample size of cervical anastomotic leakage.

The results of this study should be interpreted in the context of certain inherent limitations. It is a single-center retrospective study with a relatively small sample size. The low number of complication events and mortality limited the statistical power of the analysis of the results. Also, cellular blood components levels are dynamic and may vary in the same patient from day-to-day. Future controlled prospective studies are warranted to validate the predictive risk models.

Despite these limitations, this is the first study to assess the relationship between peripheral blood count variables changes during neoadjuvant chemoradiotherapy using a platinum- and taxane-based regimen followed by curative intent esophagectomy for esophageal cancer in predicting postoperative complications. Variables of routine blood count are already in use as part of the patient's routine laboratory work for esophageal cancer patients,

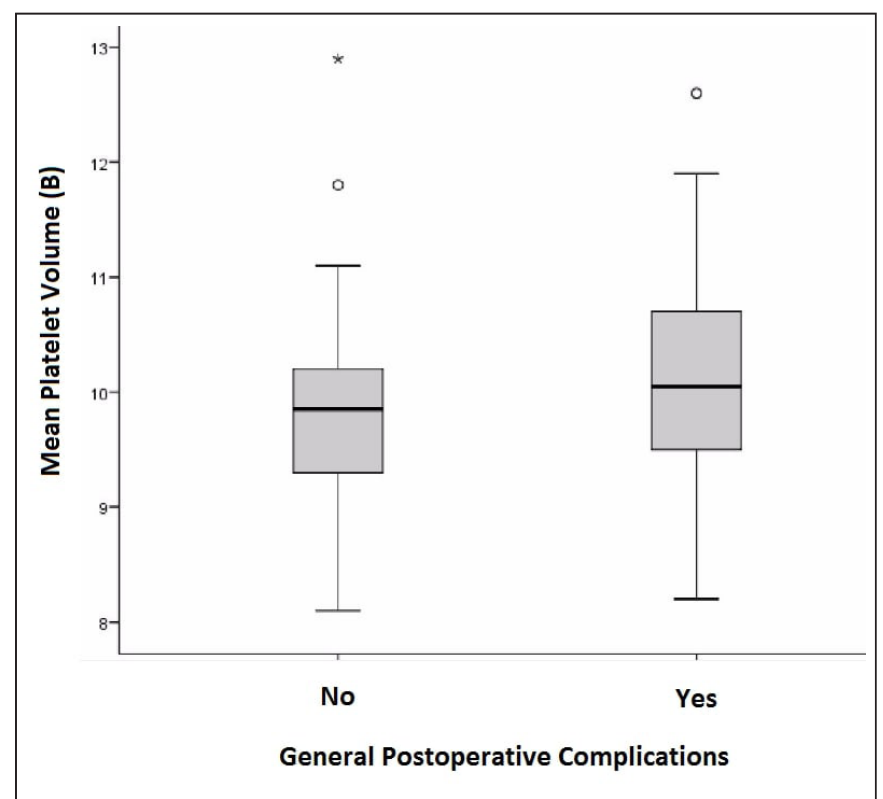

FIGURE 2. Box-plot of mean platelet volume (MPV) before surgery and its relationship with postoperative complications. MPV are expressed in $\mathrm{fL}$.

and are easily assessable, cost-effective, readily available, and can be used as predictive risk factors for postoperative outcomes.

\section{CONCLUSION}

The platelet volume prior to surgery is related to postoperative complications and the lymphocyte count change prior to surgery predicts severe postoperative complications in the setting of trimodal therapy for esophageal cancer.

\section{Authors' contribution}

Tustumi F: analysis and interpretation of data. Takeda FR: acquisition of data. Brandão AAGS: drafting the article. Sallum RAA: revising the article critically for important intellectual content. Ribeiro Junior U: conception and design of the study. Cecconello I: final approval of the version to be submitted.

\section{Orcid}

Francisco Tustumi. Orcid: 0000-0001-6695-0496.

Flávio Roberto Takeda. Orcid: 0000-0001-7338-922X.

Antonio Adolfo Guerra Soares Brandão. Orcid: 0000-00021826-611X.

Rubens Antonio Aissar Sallum. Orcid: 0000-0003-1823-0042. Ulysses Ribeiro Junior. Orcid: 0000-0003-1711-7347.

Ivan Cecconello. Orcid: 0000-0002-3535-4170. 
Tustumi F, Takeda FR, Brandão AAGS, Sallum RAA, Ribeiro Junior U, Cecconello I. Linfócitos e volume plaquetário estão associados a complicações pós-esofagectomia por câncer: um estudo de coorte. Arq Gastroenterol. 2019;56(4):377-85.

RESUMO - Contexto - Os biomarcadores obtidos do hemograma completo são fatores prognósticos a longo prazo em pacientes com câncer. No entanto, o valor desses biomarcadores no contexto da terapia trimodal para o câncer de esôfago na predição de resultados pós-operatórios precoces não é estudado. Objetivo - O presente estudo avaliou o papel dos componentes celulares do sangue na predição de mortalidade e morbidade pós-operatória. Métodos - Uma coorte de 149 pacientes consecutivos submetidos à quimiorradioterapia usando esquemas baseados em platina e taxano seguidos por esofagectomia foi analisada. Os componentes celulares do sangue coletados antes da terapia neoadjuvante (período A) e antes da cirurgia (período B) foram avaliados quanto à mortalidade e complicações pós-operatórias. Modelos de regressão de Cox univariada e multivariada foram aplicados para avaliar a significância prognóstica independente das variáveis da contagem sanguínea. Resultados - A morbidade pós-operatória esteve presente em $46 \%$ dos pacientes. Na análise de regressão múltipla, o volume plaquetário (B) (OR: 1,53; IC95\%: 1,2-2,33) foi um preditor independente de complicações gerais. Complicações cirúrgicas pós-operatórias graves estavam presentes em 17\% dos pacientes. Na análise de regressão múltipla, a diminuição de linfócitos entre os períodos B-A (OR: 0,992; 95\% CI: 0,990-0,997) esteve relacionada ao maior risco de complicações graves. Fístula da anastomose cervical esteve presente em $25,6 \%$ dos pacientes. Na análise univariada, a contagem de eosinófilos nos períodos A e B relacionou-se com a fístula da anastomose cervical. Para este resultado, o modelo multivariado de articulação não conseguiu identificar variáveis de risco independentes entre os componentes celulares do sangue. A taxa de mortalidade em 30 dias foi de 7,4\%. Na análise univariada, a contagem no período B foi associada a maior risco de mortalidade. O modelo multivariado de articulação não pôde predizer mortalidade devido ao pequeno número de pacientes no grupo de mortalidade. Conclusão - Este é o primeiro estudo a avaliar o papel das variáveis do hemograma durante a quimiorradioterapia neoadjuvante para câncer na predição de complicações pós-operatórias. Volume plaquetário e variação da contagem de linfócitos séricos antes da cirurgia podem ser utilizados como biomarcadores preditivos de complicações pós-operatórias nos pacientes com neoplasia de esôfago submetidos a terapia trimodal.

DESCRITORES - Neoplasias esofágicas. Terapia neoadjuvante. Células sanguíneas. Leucócitos. Plaquetas. Linfócitos. Neutrófilos.

\section{REFERENCES}

1. van Hagen P, Hulshof MC, van Lanschot JJ, Steyerberg EW, van Berge Henegouwen MI, Wijnhoven BP, et al. Preoperative chemoradiotherapy for esophageal or junctional cancer. N Engl J Med. 2012;366:2074-84.

2. Mota FC, Cecconello I, Takeda FR, Tustumi F, Sallum RAA, Bernardo WM. Neoadjuvant therapy or upfront surgery? A systematic review and meta-analysis of T2N0 esophageal cancer treatment options. Int J Surg. 2018;54:176-81.

3. Booka E, Takeuchi H, Nishi T, Matsuda S, Kaburagi T, Fukuda K, et al. The Impact of Postoperative Complications on Survivals After Esophagectomy for Esophageal Cancer. Medicine (Baltimore). 2015;94:e1369.

4. Hyder J, Boggs DH, Hanna A, Suntharalingam M, Chuong MD. Changes in neutrophil-to-lymphocyte and platelet-to-lymphocyte ratios during chemoradiation predict for survival and pathologic complete response in trimodality esophageal cancer patients. J Gastrointest Oncol. 2016;7:189-95.

5. Kwon HC, Kim SH, Oh SY, Lee S, Lee JH, Choi HJ, et al. Clinical significance of preoperative neutrophil-lymphocyte versus platelet-lymphocyte ratio in patients with operable colorectal cancer. Biomarkers. 2012;17:216-22.

6. Liu JS, Huang Y, Yang X, Feng JF. A nomogram to predict prognostic values of various inflammatory biomarkers in patients with esophageal squamous cell carcinoma. Am J Cancer Res. 2015;5:2180-9.

7. Miao C, Zhu S, Pan H, Cao X, Yuan S, Hu X. Combined neutrophil-platelet score and hemoglobin level predict survival in esophageal squamous cell carcinoma patients treated with chemoradiotherapy. Oncotarget. 2017:8:87971-9.

8. Rassouli A, Saliba J, Castano R, Hier M, Zeitouni AG. Systemic inflammatory markers as independent prognosticators of head and neck squamous cell carcinoma. Head Neck. 2015;37:103-10.

9. Sharaiha RZ, Halazun KJ, Mirza F, Port JL, Lee PC, Neugut AI, Altorki NK, Abrams JA. Elevated preoperative neutrophil: lymphocyte ratio as a predictor of postoperative disease recurrence in esophageal cancer. Ann Surg Oncol. 2011;18:3362-9.

10. Smith RA, Bosonnet L, Raraty M, Sutton R, Neoptolemos JP, Campbell F, Ghaneh P. Preoperative platelet-lymphocyte ratio is an independent significant prognostic marker in resected pancreatic ductal adenocarcinoma. Am J Surg. 2009; 197:466-72.

11. Walsh SR, Cook EJ, Goulder F, Justin TA, Keeling NJ. Neutrophil-lymphocyte ratio as a prognostic factor in colorectal cancer. J Surg Oncol. 2005;91:181-4

12. Wang CY, Hsieh MJ, Chiu YC, Li SH, Huang HW, Fang FM, Huang YJ. Higher serum C-reactive protein concentration and hypoalbuminemia are poor prognostic indicators in patients with esophageal cancer undergoing radiotherapy. Radiother Oncol. 2009;92:270-5
13. Cakmak Templeton AJ, McNamara MG, Seruga B, et al. Prognostic role of neutrophil-to-lymphocyte ratio in solid tumors: a systematic review and meta-analysis. J Natl Cancer Inst. 2014;106:dju124.

14. Szor DJ, Dias AR, Pereira MA, Ramos MFKP, Zilberstein B, Cecconello I, Ribeiro-Júnior U. Prognostic Role of Neutrophil/Lymphocyte Ratio in Resected Gastric Cancer: A Systematic Review and Meta-analysis. Clinics (Sao Paulo). 2018;73:e360.

15. Xiao Q, Zhang B, Deng X, Wu J, Wang H, Wang Y, Wang W. The Preoperative Neutrophil-To-Lymphocyte Ratio Is a Novel Immune Parameter for the Prognosis of Esophageal Basaloid Squamous Cell Carcinoma. PLoS One. 2016;11: e0168299.

16. Vulliamy P, McCluney S, Mukherjee S, Ashby L, Amalesh T. Postoperative Elevation of the Neutrophil: Lymphocyte Ratio Predicts Complications Following Esophageal Resection. World J Surg. 2016;40:1397-403.

17. Rice TW, Patil DT, Blackstone EH. 8th edition AJCC/UICC staging of cancers of the esophagus and esophagogastric junction: application to clinical practice. Ann Cardiothorac Surg. 2017;6:119-30.

18. Low DE, Alderson D, Cecconello I, Chang AC, Darling GE, D'Journo XB, et al. International Consensus on Standardization of Data Collection for Complications Associated With Esophagectomy: Esophagectomy Complications Consensus Group (ECCG). Ann Surg. 2015;262:286-94.

19. Dindo D, Demartines N, Clavien PA. Classification of surgical complications: a new proposal with evaluation in a cohort of 6336 patients and results of a survey. Ann Surg. 2004;240:205-13.

20. Ryan R, Gibbons D, Hyland JM, Treanor D, White A, Mulcahy HE, O'Donoghue DP, Moriarty M, Fennelly D, Sheahan K. Pathological response following long-course neoadjuvant chemoradiotherapy for locally advanced rectal cancer. Histopathology. 2005;47:141-6.

21. Paramanathan A, Saxena A, Morris DL. A systematic review and meta-analysis on the impact of pre-operative neutrophil lymphocyte ratio on long term outcomes after curative intent resection of solid tumours. Surg Oncol. 2014;23:31-9.

22. Balkwill F, Mantovani A. Inflammation and cancer: back to Virchow? Lancet. 2001;357:539-45.

23. Guthrie GJ, Charles KA, Roxburgh CS, Horgan PG, McMillan DC, Clarke SJ. The systemic inflammation-based neutrophil-lymphocyte ratio: experience in patients with cancer. Crit Rev Oncol Hematol. 2013;88:218-30.

24. Casey J, Flinn WR, Yao JS, Fahey V, Pawlowski J, Bergan JJ. Correlation of immune and nutritional status with wound complications in patients undergoing vascular operations. Surgery. 1983;93:822-7. 
25. Kubo T, Ono S, Ueno H, Shinto E, Yamamoto J, Hase K. Impact of the perioperative neutrophil-to-lymphocyte ratio on the long-term survival following an elective resection of colorectal carcinoma. Int J Colorectal Dis. 2014;29:1091-9.

26. Takeda FR, Viyuela MS, da Cruz Junior JB, Tustumi F, Braghiroli OFM, Karolyne Ernesto Luiz Nobre KEL, et al. Variables Associated to Pathologic Complete Response, Overall Survival and Disease-Free Survival in the Neoadjuvant Setting for Esophageal Cancer: A Retrospective Cohort Analysis. Int Surg: 2018;103:214-21.

27. Nowarski R, Gagliani N, Huber S, Flavell RA. Innate immune cells in inflammation and cancer. Cancer Immunol Res. 2013;1:77-84.

28. Alexandrakis MG, Passam FH, Moschandrea IA, Christophoridou AV, Pappa CA, Coulocheri SA, Kyriakou DS. Levels of serum cytokines and acute phase proteins in patients with essential and cancer-related thrombocytosis. Am J Clin Oncol. 2003;26:135-40.

29. Yun ZY, Li N, Zhang X, Zhang H, Bu Y, Sun Y, et al. Mean platelet volume, platelet distribution width and carcinoembryonic antigen to discriminate gastric cancer from gastric ulcer. Oncotarget. 2017;8:62600-5.

30. Grivennikov SI, Greten FR, Karin M. Immunity, inflammation, and cancer. Cell. 2010;140:883-99.

31. Zhao W, Wang P, Jia H, Chen M, Gu X, Liu M, Zhang Z, Cheng W, Wu Z. Lymphocyte count or percentage: which can better predict the prognosis of advanced cancer patients following palliative care? BMC Cancer. 2017;17:514

32. Dutta S, Crumley AB, Fullarton GM, Horgan PG, McMillan DC. Comparison of the prognostic value of tumour- and patient-related factors in patients undergoing potentially curative resection of oesophageal cancer. World J Surg. 2011;35:1861-6.

33. Nishida T, Sakakibara H. Association between underweight and low lymphocyte count as an indicator of malnutrition in Japanese women. J Womens Health (Larchmt). 2010;19:1377-83.

34. Bergquist J, Tarkowski A, Ewing A, Ekman R. Catecholaminergic suppression of immunocompetent cells. Immunol Today. 1998;19:562-7.

35. Weigent DA. Hypoxia and cytoplasmic alkalinization upregulate growth hormone expression in lymphocytes. Cell Immunol. 2013;282:9-16.

36. Mackall CL, Fleisher TA, Brown MR, Magrath IT, Shad AT, Horowitz ME, et al. Lymphocyte depletion during treatment with intensive chemotherapy for cancer. Blood. 1994;84:2221-8.

37. Aghdaii N, Ferasatkish R, Mohammadzadeh Jouryabi A, Hamidi SH. Significance of preoperative total lymphocyte count as a prognostic criterion in adult cardiac surgery. Anesth Pain Med. 2014;4:e20331.

38. Bian C, Wu Y, Shi Y, Xu G, Wang J, Xiang M, Weng S, et al. Predictive value of the relative lymphocyte count in coronary heart disease. Heart Vessels. 2010;25:469-73.

39. Major AS, Fazio S, Linton MF. B-lymphocyte deficiency increases atherosclerosis in LDL receptor-null mice. Arterioscler Thromb Vasc Biol. 2002;22:1892-8.
40. Núñez J, Sanchis J, Bodí V, Núñez E, Mainar L, Heatta AM, et al. Relationship between low lymphocyte count and major cardiac events in patients with acute chest pain, a non-diagnostic electrocardiogram and normal troponin levels. Atherosclerosis. 2009;206:251-7.

41. Mohri Y, Tanaka K, Toiyama Y, Ohi M, Yasuda H, Inoue Y, Kusunoki M. Impact of Preoperative Neutrophil to Lymphocyte Ratio and Postoperative Infectious Complications on Survival After Curative Gastrectomy for Gastric Cancer: A Single Institutional Cohort Study. Medicine (Baltimore). 2016;95:e3125.

42. Shiraishi Y, Fang P, Xu C, Song J, Krishnan S, Koay EJ, Mehran RJ, et al. Severe lymphopenia during neoadjuvant chemoradiation for esophageal cancer: A propensity matched analysis of the relative risk of proton versus photon-based radiation therapy. Radiother Oncol. 2018;128:154-60.

43. Josse JM, Cleghorn MC, Ramji KM, Jiang H, Elnahas A, Jackson TD, et al. The neutrophil-to-lymphocyte ratio predicts major perioperative complications in patients undergoing colorectal surgery. Colorectal Dis. 2016;18:O236-42.

44. Nozoe T, Kimura Y, Ishida M, Saeki H, Korenaga D, Sugimachi K. Correlation of pre-operative nutritional condition with post-operative complications in surgical treatment for oesophageal carcinoma. Eur J Surg Oncol. 2002;28:396-400.

45. Maruyama Y, Inoue K, Mori K, Gorai K, Shimamoto R, Onitsuka T, et al. Neutrophil-lymphocyte ratio and platelet-lymphocyte ratio as predictors of wound healing failure in head and neck reconstruction. Acta Otolaryngol. 2017;137:106-10

46. Kang WM, Zhu CZ, Yang XX, Yu JC, Ma ZQ, Ye X, et al. Application of the Onodera prognostic nutrition index and neutrophil-to-lymphocyte ratio in risk evaluation of postoperative complications in Crohn's disease. Sci Rep. 2017;7:8481.

47. Galdiero MR, Varricchi G, Seaf M, Marone G, Levi-Schaffer F, Marone G. Bidirectional Mast Cell-Eosinophil Interactions in Inflammatory Disorders and Cancer. Front Med (Lausanne). 2017;4:103.

48. Lotfi R, Lee JJ, Lotze MT. Eosinophilic granulocytes and damage-associated molecular pattern molecules (DAMPs): role in the inflammatory response within tumors. J Immunother. 2007;30:16-28.

49. Ito T, Hirahara K, Onodera A, Koyama-Nasu R, Yano I, Nakayama T. Anti-tumor immunity via the superoxide-eosinophil axis induced by a lipophilic component of Mycobacterium lipomannan. Int Immunol. 2017;29:411-21.

50. Sakkal S, Miller S, Apostolopoulos V, Nurgali K. Eosinophils in Cancer: Favourable or Unfavourable? Curr Med Chem. 2016;23:650-66.

51. Uemura K, Nakajima M, Yamauchi N, Fukayama M, Yoshida K. Sudden death of a patient with primary hypereosinophilia, colon tumours, and pulmonary emboli. J Clin Pathol. 2004;57:541-3.

52. Aderka D, Brown A, Zelikovski A, Pinkhas J. Idiopathic deep vein thrombosis in an apparently healthy patient as a premonitory sign of occult cancer. Cancer. $1986 ; 57: 1846-9$ 